\title{
Die "diabetische“ Herzinsuffizienz hat viele Ursachen
}

\author{
Der Typ-2-Diabetes mellitus ist eine der häufigsten Komorbiditäten bei Patienten mit \\ einer chronischen Herzinsuffizienz, andererseits entwickeln Typ-2-Diabetiker auch sehr \\ viel häufiger eine Herzinsuffizienz als Stoffwechselgesunde. Pathogenetisch spielen die \\ Hypertonie und die KHK die entscheidende Rolle, aber auch unabhängig von diesen \\ Begleiterkrankungen entwickelt sich nicht selten eine diabetische Kardiomyopathie.
}

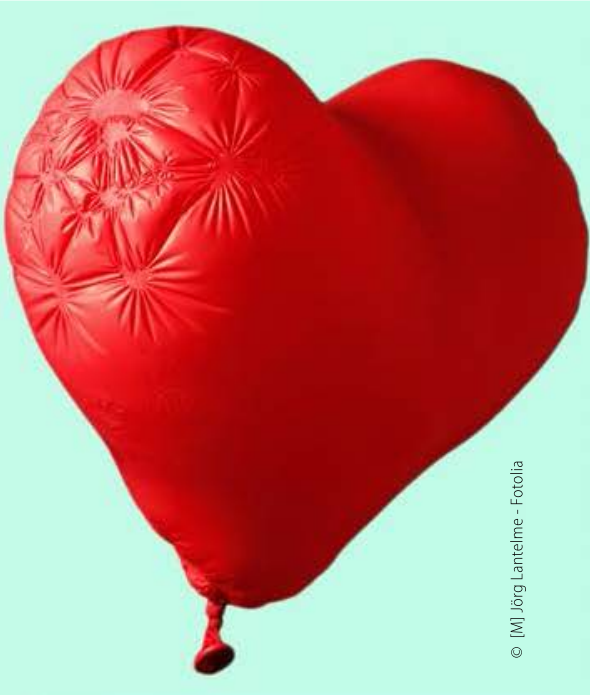

„Obwohl die Herzinsuffizienz eine der häufigsten und gefährlichsten Begleiterkrankungen bzw. Komplikationen des Typ2-Diabtets ist, wird diesem Krankheitsbild in der Forschung und auch im klinischen Alltag zu wenig Aufmerksamkeit geschenkt“, erläuterte Prof. Eberhard Standl, München.

Die Diagnose Herzinsuffizienz wird nach den Ergebnissen epidemiologischer Studien bei Typ-2-Diabetikern bis zu 5-mal häufiger gestellt als bei Stoffwechselgesunden. Auch manifestiert sie sich bei Diabetikern früher als bei Nichtdiabetikern. Das Risiko für eine notfallmäßige stationäre Aufnahme wegen Herzinsuffizienz ist bei Diabetikern um 33\% höher als bei Stoffwechselgesunden. Und die Manifestation geht mit einer deutlich schlechteren Prognose einher und umgekehrt verschlechtert das Vorliegen eines Typ-2-Diabetes die Überlebenschance bei Herzinsuffizienz, und zwar sowohl bei der systolischen als auch bei der diastolischen. So haben herzinsuffiziente Typ-2-Diabetiker ein 12-fach erhöhtes Mortalitätsrisiko im Vergleich zu Diabetikern ohne Herzinsuffizienz und ein 2bis 3-fach höheres kardiovaskuläres Sterberisiko als Stoffwechselgesunde. „Daraus ergibt sich die zwingende Notwendigkeit einer engen Zusammenarbeit zwischen Diabetologe und Kardiologe", so Standl. Bei kardiologischen Patienten mit KHK oder Herzinsuffizienz sollte immer nach einem Diabetes mellitus gefahndet werden und umgekehrt sollte ein Typ-2-Diabetiker auch kardiologisch mittels Echo und Ergometrie abgeklärt werden [1].

\section{Multifaktorielles Geschehen}

Die Pathogenese der diabetischen Herzinsuffizienz ist komplex. Die häufigsten Ursachen sind die KHK und arterielle Hypertonie. Aber auch unabhängig von beiden Erkrankungen kommt es bei Typ-2-Diabteikern zu einer myokardialen Schädigung, man spricht von einer diabetischen Kardiomyopathie [2]. „Sie ist das Ergebnis verschiedener Mechanismen“, so Standl. Dazu gehören metabolische Einflüsse wie Hyperglykämie, Hyperinsulinismus und ein Anstieg der freien Fettsäuren, die toxisch für das Myokard sind, und interzelluläre Einlagerungen bzw. die verstärkte Bildung von interstitiellem Gewebe i.S. einer Fibrosierung ebenso wie eine autonome Dysfunktion mit inadäquater Frequenzregulation, eine endotheliale Dysfunktion und mikrovaskuläre Veränderungen. Folge ist eine veränderte myokardiale Energetik bzw. ein prolongiertes relatives Energiedefi- zit $[3,4]$. „Keine dieser Veränderungen ist spezifisch für Diabetes, aber beim Diabetiker kommen viele Dinge zusammen", so Standl. Folgen sind strukturelle und funktionelle Störungen i.S. einer diastolischen oder systolischen Herzinsuffizienz.

\section{Welche Antidiabetika sind unproblematisch}

Die Behandlung der diabetischen Kardiomyopathie unterscheidet sich nicht von der bei anderen Formen der Herzinsuffizienz. In den entsprechenden Studien erwiesen sich ACEHemmer bzw. AT1-Blocker und Betablocker bei Typ-2-Diabetikern aber als besonders effektiv. Die Therapie sollte sich deshalb an diesen Leitlinien orientieren. Ein wichtiger Prädiktor für die Prognose bei herzinsuffizienten Diabetikern ist die Insulinresistenz und der Bauchumfang, woraus sich auch Möglichkeiten zur Prävention ergeben. In der UKPDS konnte gezeigt werden, dass eine Senkung des $\mathrm{HbA}_{1 \mathrm{c}}$-Wertes um $1 \% \mathrm{zu}$ einer signifikanten Abnahme des Risikos für die Entwicklung einer Herzinsuffizienz führt. „Alle Faktoren , die den Diabetes begünstigen, begünstigen auch die Herzinsuffizienz", so Standl. Dagegen hätten neuere Studien gezeigt, dass eine zu starke glykämische Kontrolle durch Medikamente die Prognose sogar verschlechtere, sehr wahrscheinlich als Folge von Hypoglykämien.

Was die antidiabetische Medikation betrifft, so sind Pioglitazon, Gliptine und auch GLP-1-Analoga im Hinblick auf das Herz unproblematisch. Bei schwerer Herzinsuffizient sollte aber kein Metformin eingesetzt werden. Und in der EMPA-REG OUTCOMETM-Studie konnte erstmals für ein Antidiabetikum, nämlich für Empagliflozin, eine günstige Beeinflussung der Prognose und auch der Notwendigkeit für eine stationäre Behandlung wegen Herzinsuffizienz dokumentiert werden [5].

Dr. med. Peter Stiefelhagen

\author{
Literatur: \\ 1. Halle M et al. DMW 137: 437-41. \\ 2. Stratmann B et al. Diabetes Care 34 (Suppl 2): 138-44. \\ 3. Pappachan J M et al. World J Diabetes 4 (2013): 177-89. \\ 4. Niemann M et al. Herz 38 (2013): 41-7. \\ 5. Zinman B et al. N Engl J Med 2015; DOI: 10.1056/NEJMoa1504720
}

Quelle: 8th Annual Meeting of the Diabetes and Cardiovascular Disease EASD Study Group and 1st CVOT Summit in Diabetes, 31.10.2015 in Unterschleissheim bei München 